superseding divisive alliances have barely outlived that decade; but the recently renascent concept of 'human security' 28 teaches that privileged regions like Europe should focus on helping poorer communities that lack security in every dimension - i.a. by cutting back on their static military investments to re-direct resources towards civilian reaction capacities and crisis prevention. Similar conclusions may be reached from a different angle by those who do not want the EU to become 'militarized' or the life of European populations to be any more 'securitized' than at present. ${ }^{29}$ On this view, even if there is some 'dirty business' of collective defence still to be done, the EU should leave others to do it and should certainly not draw military forces and assets any further into its own internal security tasks. At stake are not only the EU's predominately civilian traditions and peaceful norms, but also its image and legitimacy as seen by the rest of the world.

It is not the aim of this article to judge such positions but rather, to suggest that they deserve further debate and testing against the main line of argument developed above. It can hardly be denied that security realities will continue to invade European

28 See for example 'A Human Security Doctrine for Europe: Report of the Study Group on Europe's Security Capabilities', published on 15 Sep. 2004 at http://www.lse.ac.uk/Depts/global/\#Recent\%20contributions\%20by\%20our\%20staff. 29 This is a widely held view, for example, in Sweden, Denmark and Ireland. societies, whether in the form of deadly terrorism or of equally deadly epidemics, violent storms, energy black-outs or the deaths of hundreds of citizens on holiday abroad. In some sense, in the globalized world and the frontier-free Europe, all citizens are now exposed to the kind of risks and - potentially - the personal responsibilities to show discipline and help the vulnerable that soldiers take on for the temporary term of their service. The greater specialization of the soldier's function does not, therefore, need to carry him/her further away from the ordinary citizen in normative and experiential terms: and the question of how to make the soldier a good citizen need be no less relevant for Europe today than it was in 1945. Of course, if this need and the EU's potential role in meeting it should be accepted, a whole new range of questions for research and for policy would arise. History itself prevents an EU 'collective defence' culture being built on the same basis as NATO's in the 1950's. Should we look to 'Europeanize' our soldiers today by bottom-up functional integration and common weaponry, or by a common mission to protect their common homelands against the new spectrum of threats, or by stringent common norms that will make Europe proud of them when operating abroad? The most likely answer would be a combination of all of these, plus some surprises that the next phase of history no doubt holds in store for us yet.

\title{
Autoritäre Demokratisierung in Usbekistan
}

\author{
Hendrik Fenz*
}

\begin{abstract}
The first globalisation decade began in 1992, when the country joined the International Monetary Fund and the World Bank. Full of optimism, Uzbekistan started into independence trying to reform under the flag of democracy and rule of law. It has reformed, however, without giving up experienced mechanisms of authoritarian rule and corruption from past decades. With this system-immanent contradiction, the country is an obstacle to its own political development. Under the banner of the anti-terror struggle, Uzbek leaders already in 1998 started a disastrous campaign against Islamic believers, who were striving for independence from the state-controlled religion practice. Not only Islamic extremists fell victim to the campaign but mostly ordinary Muslims. Consequently, the country seems to manoeuvre into political imbalance, more and more leaving democratic standards.
\end{abstract}

Keywords: Autoritarismus, Demokratisierung, Islamismus, Usbekistan

\section{Die usbekische Version von Good Governance: Autokratie}

$\mathrm{U}$ sbekistan ist Daewoo-Land. Nicht deutlicher könnte sich die Globalisierung in Usbekistan zeigen, als auf den Straßen. An verrosteten und Jahrzehnte alten Sowjetmobilen geht es mit koreanischen Daewoos auf der Überholspur in die neue Zukunft. Zwei Modelle prägen das Bild: ein microkleiner 6-Sitzer, der nicht selten als Sammeltaxi

* Dr. Hendrik Fenz, Turkologe, wissenschaftlicher Mitarbeiter am AsienAfrika-Institut der Universität Hamburg. unterwegs ist, und eine flotte Limousine, die selbst bei 140 $\mathrm{km} / \mathrm{h}$ den usbekischen Straßen zu trotzen vermag. Wer die 6.000 Euro für die kleine Version aufbringen kann, darf sich zu den Gewinnern des gesellschaftlichen Wandels zählen. An einem Lehrergehalt gemessen, das 2003 bei ca. 30 Euro lag, dürften sich diesen Luxus allerdings nicht viele Familien leisten können.

Ein Großteil der Bevölkerung lebt unterhalb der 1-DollarArmutsgrenze. Viele Indikatoren im aktuellen HumanDevelopment-Report der UNO verweisen Usbekistan auf die unteren Plätze: Ob Kindersterblichkeit, Analphabetismus oder 
Lebenserwartung - dem Land geht es nachweisbar schlechter als zu Sowjetzeiten. ${ }^{1}$

Der Schadensanalysen gibt es viele und je nach politischem Standpunkt machen sie den sowjetischen Sozialismus, politischen Islamismus oder die Globalisierung für den gesellschaftlichen Niedergang verantwortlich. Die eigentlichen Ursachen liegen neben dem wirtschaftlichen Desaster im Inneren. Der Umgang mit der Opposition, angefangen bei nationalen, antirussischen Organisationen der Frühphase der Transition, bis hin zu islamistischen Radikalen, war und ist gekennzeichnet von Restriktionen und Verboten bis hin zu bewaffneten Aktionen. Der geringe politische Spielraum brachte gerade jene Radikalisierung mit sich, welche die Regierung zu vermeiden bestrebt war.

Das Aufbrechen sozialistischer Herrschaftsstrukturen - soweit das Fazit zur ersten Globalisierungsdekade - mündete nicht in Demokratie und Rechtsstaatlichkeit. Nur wenige Länder der »Früheren Sowjetunion« (FSU), etwa Weißrussland und Turkmenistan, verfügen gegenwärtig über eine solch manifeste und auf den Staatspräsidenten ausgerichtete Machtpyramide wie Usbekistan. Während das demokratische Transitionspaket aus Zivilgesellschaft und Good Governance fest zugeschnürt bleibt und damit auch Pluralismus, Meinungs-, Glaubens- und Pressefreiheit staatlichem Diktat unterliegen, feiert ein präsidialer Personenkult weitgehend ungehindert fröhliche Urstände. Präsident Islam Karimov (geboren 1938), noch zu Sowjetzeiten zum Präsidenten Usbekistans gewählt, gehört zu den politischen Gewinnern der Transition. Die Zähigkeit sowjetischer Netzwerke zeigt sich nicht zuletzt in der Formierung einer neuen, für das moderne Usbekistan unbekannten Herrschaftsform: der »Erbdemokratur «. Diese lässt sich definieren als der Versuch, entlang demokratischer Insignien generationsübergreifende Herrschaftssicherung zu betreiben. Im Übrigen eine Tendenz, die auch in anderen FSU-Staaten zum Tragen kommt. Namentlich in Aserbaidschan wanderten Zepter und Krone im Herbst 2003 problemlos vom Vater auf den Sohn, vom Präsidenten Haydar Aliyev zum damals amtierenden Ministerpräsidenten Ilham Aliyev. ${ }^{2}$

Mit der Unterdrückung jeglicher politischer Opposition verbaut der Staat nicht nur den demokratieorientierten Organisationen den Weg in die Mitbestimmung, sondern ebnet damit - ungewollt aber konfliktbehaftet - radikalen Gruppierungen den Zugang zur desillusionierten Bevölkerung.

Es ist daher kaum ein Wunder, dass vor allem islamistische Organisationen wie die Islamische Bewegung Usbekistans (IBU) und die Hizb ut Tahrir al-Islami (HUT) im Land Fuß fassen konnten. Der Aktionsradius legaler und staatlich registrierter Organisationen hingegen bleibt auch nach über einem Jahrzehnt Transition gering. Politische Parteien spielen - so sie überhaupt ein real existierendes Potential haben - von der Regierungspartei geschriebene Nebenrollen. So zählt das Abschneiden von Abdulhafiz Djalalov, Chef der Volksdemokratischen Partei Usbekistans (VDP), in den Präsidentschaftswahlen 2000 als

1 Vgl.: www. undp.org/hdr2003/german/pdf/hdr03_ge_complete.pdf (Zugriff am 15. Januar 2004)

2 Ilham Aliyev sitzt als Vizepräsident des staatlichen Ölkonzerns SOCAR an finanzkräftiger Quelle, um die Regierungsmaschine auch in Zukunft kräftig schmieren zu können.
Sieg der Demokratie. Die erzielten 4,17 \% stellten »einen realen Beitrag dieser größten und einflußreichsten politischen Partei zum Aufbau des demokratischen Wahlverfahrens in Usbekistan dar«. Im gleichen Atemzug werden die 91,9 \% für Islam Karimov als deutliches Zeichen für die Unterstützung des Reformkurses des Präsidenten gewertet. ${ }^{3}$ Nach Interpretation der Zentralen Wahlkommission Usbekistans scheinen internationale Wahlbeobachtermissionen, etwa die OSZE, dem Recht zu geben: Die Wahlen hätten internationalen Standards entsprochen und hätten in Übereinstimmung mit den Rechtsgrundlagen stattgefunden. Schaut man sich einmal OSZE-Berichte direkt an, kommen Zweifel auf an den Auslegungen der Wahlkommission; zeigen sich im Original doch reihenweise Kritikpunkte, die vom Aushebeln des Versammlungsrechtes bis hin zur Behinderung von Kandidaten reichen. ${ }^{4}$ Die Regierung selbst verliert kein Wort über die Behinderungen der Opposition oder über die Zensur von Zeitungen und Zeitschriften. Human Rights Watch (HRW) kritisiert denn auch die punktuellen Verbesserungen als unverbindliche Einzelfälle und nicht richtungsweisend für einen politischen Wandel: »Human rights abuses on a massive scale continued in Uzbekistan in 2002. The closer relationship with the United States (U.S.) that developed after the September 11, 2001 terrorist attacks on the U.S. - including the provision of air bases for U.S. troops - pushed Uzbekistan to make some gestures to show progress on human rights. However, these did not amount to any fundamental improvement. The government systematically violated the rights to freedom of religion, expression, association, and assembly. There was no independent judiciary, and torture was widespread in both pre-trial and post-conviction facilities. $\ll^{5}$

Immerhin ließen sich seitdem Verbesserungen im Umgang mit der nationalen Opposition verzeichnen. Es ist eher eine »Politik der kleinen Schritte « und ein pragmatisches Eingeständnis in die eigenen Grenzen der Repressionspolitik, die den Präsidenten Anfang 2000 zum Aufheben der Zensur verleiteten. Ein Hauch von Zivilgesellschaft machte sich breit. Daraus eine politische Tendenz abzuleiten, wäre jedoch allzu optimistisch. Die über 450 NGOs in Usbekistan simulieren ein reges politisches Leben: Ihrem Bewegungs- und Artikulationsrahmen sind jedoch enge Grenzen gesetzt. Selbst Abdulhafiz Djalalov redet der Autokratie das Wort, indem er davon spricht, dass »autoritäre Systeme häufig als Zwischenglieder beim Übergang einer Gesellschaft vom Totalitarismus zur Demokratie« fungieren. Und so begründet er denn auch gleich die Notwendigkeit einer »Politik der harten Hand «: »Der Präsident ist Staatsoberhaupt und zugleich Vorsitzender des Ministerkabinetts, also Chef der ausführenden Gewalt. [...] Man muss allerdings zugleich berücksichtigen, dass der harte und konsequente Kurs der politischen Führung gerade auf die Überwindung der Reste der totalitären Vergangenheit und der überholten autoritären Traditionen ausgerichtet ist.«

Mit anderen Worten: mit einem modernen Autoritarismus gegen den traditionellen Autoritarismus! Diese »Saat wird zweifellos

\footnotetext{
3 Vgl.: Pressedienste der Zentralen Wahlkommission und des Außenministeriums der Republik Usbekistan: www. uzbekistan.de/president4.htm (Zugriff am 10. Januar 2004).

4 Vgl.: OSCE/ODIHR: www. osce.org/odihr/?page=elections\&div=reports (Zugriff 10. Dezember 2004).

5 Vgl.: HRW: www. hrw.org/wr2k3/europe16.html (Zugriff am 10. Dezember 2004).
} 
lebensfähige Keime der Demokratie auf unserem Heimatboden sprießen lassen [...]. $\ll^{6} \mathrm{Ob}$ jedoch ein tatsächlicher Wandel auch für die Bevölkerung spürbar wird, hängt im hohen Maße von realer Machtpartizipation ab. Die gegenwärtigen strukturellen Defizite fördern eine systemische Gewalt, die sich mit demokratischen Insignien den Anschein von Legitimität verleiht. Primär gilt es, eine Rechtssicherheit herzustellen, die politische Mitsprache nicht bestraft und wirtschaftliche Investitionen berechenbar macht. Erst mit dieser Rechtssicherheit, die für Klein- und Mittelständische Unternehmen (KMU) existentiell ist und für große, auch internationale Unternehmen, langfristigen Investitionsschutz bedeutet, kann das Land auf einen wirtschaftlichen Aufschwung hoffen. Von einem politischen Systemwechsel kann in Usbekistan keine Rede sein, allenfalls von einem Dogmenwechsel. Nicht mehr der entwickelten sozialistischen Gesellschaft wird entgegengestrebt, sondern einem Staatskapitalismus; nicht mehr der egalisierende sozialistische Wettbewerb ist wirtschaftlicher Stimulator, sondern Profitmaximierung.

\section{Die Wirtschaft zwischen Kollaps, Korruption und ökologischer Katastrophe}

Unternehmen haben es schwer in Usbekistan. Sie haben nicht nur mit den rudimentären marktwirtschaftlichen Strukturen zu kämpfen, sondern ebenso mit einer für FSU-Staaten typischen Korruption. Auf dem Korruptionsindex von Transparency International firmiert Usbekistan auf Platz 114 der 145 untersuchten Staaten. ${ }^{7}$ Gesetzliche Auflagen verhindern zudem einen effizienten und direkten Zugang zum Markt. Massive Restriktionen für den Import, eine Remonopolisierung des Handels und dessen staatliche Kontrolle dämpfen in starkem Maße die Freude am Unternehmertum.

Abbildung 1: wirtschaftliche Entwicklung in einigen FSU-Staaten

Zum Vergleich: Deutschland liegt auf Platz 15. Vgl.: Transparency International, Jahresreport 2004: www. transparency.org (Zugriff am 10. Januar 2005).

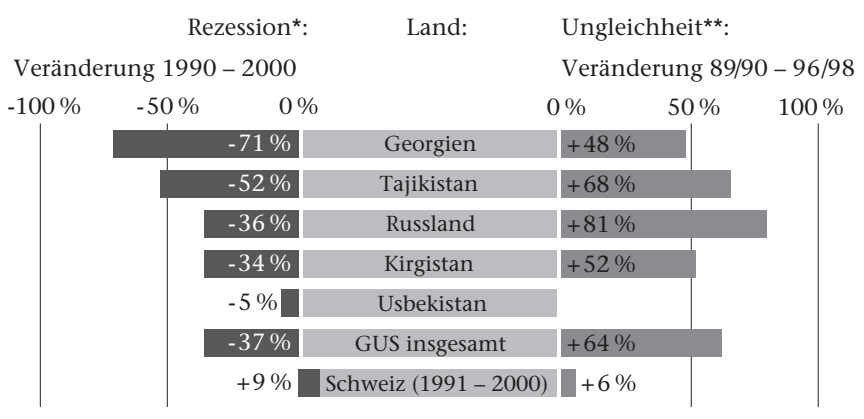

${ }^{*}$ Gemessen am realen Bruttoinlandsprodukt

**Zunahme Gini-Koeffizient (Masszahl für die Ungleichheit der Pro-Kopf-Einkommen) Hinweis: Deutschland, Wirtschaftskrise 1930/32, Einbruch der Produktion um -16\%

Quelle: www.gersterconsulting.ch

6 Abdulhafiz Djalalov: Nationale Identität unterstützt Demokratisierung, in Wostok 1-1999, S. 16

7 Zum Vergleich: Deutschland liegt auf Platz 15. Vgl.: Transparency International, Jahresreport 2004: www. transparency.org (Zugriff am 10. Januar 2005).
Mehr Freiheit, weniger Wohlstand, mehr Ungleichheit ist die höchst zwiespältige Bilanz der ersten zehn Jahre Übergang («Transition») der ehemaligen Sowjetunion von der Planzur Marktwirtschaft. Der politische Zusammenbruch war gefolgt von einer massiven Rezession in allen Ländern der die Sowjetunion ablösenden Gemeinschaft Unabhängiger Staaten (GUS), welche sogar die Weltwirtschaftskrise der 1930er Jahre in den Schatten stellt. Während die Schweiz ihre Produktion trotz des wirtschaftlichen Einbruchs der 1990er Jahre insgesamt um neun Prozent steigern konnte, sackten die GUS-Staaten zwischen 1990 und 2000 um über ein Drittel (37\%) ab. Auch der Graben zwischen Arm und Reich vertiefte sich dramatisch und transformierte die relativ egalitären Gesellschaften in solche großer Ungleichheit.

Die im großen Stil gepflegte Baumwollproduktion verbindet all jene Aspekte der Misswirtschaft sinnbildlich miteinander. Offizielle usbekische Daten sehen Usbekistan als weltweit viertgrößten Baumwollproduzenten und als zweitbedeutendsten Exporteur. ${ }^{8}$ Allerdings machen ausufernde staatliche Subventionen der US-Regierung für die einheimischen Produzenten, aber auch billige Baumwolle von afrikanischen Anbietern, etwa aus Burkina Faso, den Markt eng für Usbekistan. So ist die Produktion der Monokultur, seit Jahrzehnten das erste Exportgut Usbekistans, für den Weltmarkt zu kostenintensiv. Die regressive wirtschaftliche Lage des größten Abnehmers von usbekischer Baumwolle, Russland, trägt ebenfalls zur Stagnation bei. Ein kontinuierlicher Rückgang der Baumwollproduktion ist die Folge. Machte Baumwolle 1994 noch 47\% des Exporterlöses aus, so sind es gegenwärtig nur noch $30 \% .{ }^{9}$ Hinzu kommt ein gravierendes ökologisches Problem, das von internationalen Organisationen als die derzeit größte globale Umweltkatastrophe eingeschätzt wird: die Austrocknung des Aralsees. Der vor wenigen Jahrzehnten noch viertgrößte See der Erde ist von $64.000 \mathrm{~km}^{2}$ auf ca. $25.000 \mathrm{~km}^{2}$ eingetrocknet, der Salzgehalt hingegen von ehemals $10-14 \%$ auf ca. 36\% angestiegen. Artensterben, Verwüstung und heftige Salzstürme, die auch entfernte Gebiete erreichen und fruchtbare Böden vernichten, sind die eine Seite des Desasters. Zur anderen Seite gehören veränderte Krankheitsbilder, Missbildungen an Neugeborenen und eine noch unter dem usbekischen Durchschnitt liegende Lebenserwartung.

Dabei ist die Angst vor dem ökologischen Showdown auch den Einheimischen anzusehen. Eine Fahrt von Nukus, der 200 Kilometer entfernten Hauptstadt des autonomen Gebietes Karakalpakistan, zum Aralsee geht mit den eindringlichen Warnungen einher, ja nichts vor Ort zu konsumieren, weder dort zu essen noch zu trinken. Trotz dieser Warnung: Es leben und arbeiten Menschen in der Stadt Muynak, die früher eine industrielle Hafenstadt war, heute aber rund zwei Autostunden vom Aralsee entfernt liegt. So laut die ökologische Zeitbombe Aralsee auch ticken mag, ein generelles Umdenken auf politischer Entscheidungsebene ist nicht zu erkennen. Zwar gibt es internationale Organisationen die Renaturierungsprojekte durchführen oder initiieren. Damit wird aber lediglich an den Symptomen herumlaboriert. Dem eigentlichen Übel, der

8 Vgl.: www. uzbekistan.de/de/P_Geostrategische_Potential.htm (Zugriff am 05. Januar 2005)

9 Vgl.: www. lrp.de/m6_ausland/download/m612usb.pdf (Zugriff am 02. Januar 2004). 
Wasservernichtung durch den Baumwollanbau, gehen diese Projekte nicht an die Wurzeln. Seit Jahrtausenden speisten die Flüsse Amudarja und Syrdarja den abflusslosen Aralsee. Noch vor 25 Jahren gab es ausgedehnte Feuchtgebiete, durch deren Gräser der Amurtiger streifte.

Folgt man heute dem Amudarja auf seinem Weg vom Pamirgebirge zum Aralsee, sieht man ihn auf seinen 2.500 Kilometern von einem gewaltigen Strom zu einem kümmerlichen Rinnsal werden, dem die Kraft geraubt wurde, den Aralsee überhaupt noch zu erreichen. Seine spärlichen Reste verdunsten in der Wüste, knapp 100 Kilometer vor dem See. Daran wird sich auch in den nächsten Jahren nichts ändern. Baumwolle wird weiterhin - schon aus Mangel an Alternativen - angebaut werden. Und es wird damit das kostbare Wasser verbraucht, das für die ökologische Stabilisierung einer Region in der Größe der Bundesrepublik nötig wäre.

\section{3. Öl: Schmiermittel der Globalisierung}

Er wird als das neue »Great Game« bezeichnet: Der Kampf um die Vormachtstellung in Zentralasien und der Run aufs Erdöl. Dabei sind die Reserven an Öl und Gas bei weitem nicht so groß, wie vor Jahren gehofft. Dem Optimismus der 1990er Jahre, als noch mit Milliarden Dollar in vermeintliche Ölfelder investiert wurde, ist ein Pragmatismus gefolgt, der zwischen dem Anti-Taliban-Kampf in Afghanistan und weltwirtschaftlichen Interessen zu verorten ist. Gerade die westlichen Industriestaaten sind in den kommenden zwei Jahrzehnten mit einem wachsenden Energieversorgungsproblem konfrontiert. Die Diskrepanz zwischen Förderung und Verbrauch von Erdöl steigt kontinuierlich. Zunehmend werden Regionen wie die USA und Europa auf erhöhte Netto-Importe zurückgreifen müssen. So liegt die Importabhängigkeit der USA gegenwärtig bei $50 \%$, die Europas bei 60\%. Oder anders ausgedrückt: Die Lebensdauer der Öl-Reserven (der Quotient von Reserven zur Produktion) liegt in den USA und Kanada bei 9,5 Jahren, in Europa bei nur 7,5 Jahren, in Russland hingegen schon bei 20 und in den Golfstaaten der OPEC bei 89 Jahren. ${ }^{10}$ Die Abhängigkeit von den Golfstaaten wird sich demnach vergrößern. Umso wichtiger scheinen den USA daher die Alternativen, die sie im kaspischen und zentralasiatischen Raum pflegen und in Afrika zu suchen beginnen.

Vom Ölsektor zu unterscheiden ist das Gasgeschäft. Während es beim Öl um einen Weltmarkt geht, handelt es sich bei Gas um mehrere globale Teilmärkte. Der Transport von Erdgas ist an die Pipeline gebunden und bleibt unter gegenwärtigen Bedingungen nur bis zu einer Strecke von 6.000 Kilometern rentabel. Die riesigen Gasvorkommen, die vor allem in Russland, am Golf und im südkaspischen Raum liegen, sind interessant für Europa, kaum aber für die USA. Der globale Teilmarkt Gas im euro-asiatischen Raum berührt daher vor allem Europa, das schon jetzt zum überwiegenden Teil sein Erdgas aus diesen Gebieten bezieht. Der mit den zentralasiatischen Staaten im September 1997 abgeschlossene Energiecharta-Vertrag be-

10 Vgl.: Müller, Friedemann: Energiepolitische Interessen in Zentralasien, in: www. Bundestag.de/cgi-bin/druck.pl?N=parlament (Zugriff am 08. Februar 2003). stimmt verbindlich die Zusammenarbeit bei der Erschließung der Öl- und Gasfelder sowie bei der Streckenführung von Pipelines. ${ }^{11}$ Die von der EU initiierten Programme Inogate, Traceca und Tacis sind weitere Bausteine im System der europäischen Förderung des euro-asiatischen Wirtschaftsraumes. Umso mehr, als bei schwindenden europäischen Reserven und steigenden Netto-Erdgasimporten verstärkt auf die euroasiatischen Märkte zurückgegriffen werden muss. Das damit steigende Abhängigkeitsverhältnis steht jedoch, trotz aller europäischer Maßnahmen, kaum im Verhältnis zu der politischen Zurückhaltung. Dafür sind die europäischen Binnenstrukturen verantwortlich. Unklare Entscheidungswege und nationale Befindlichkeiten schaffen ein Kompetenzvakuum. Gefragt ist daher eine Politik, die einen euro-asiatischen PipelineVerbund fördert, Zentralasien ebenso einschließt wie die Türkei, Bulgarien und Rumänien. Dabei wird der Beitrag Usbekistans an der Weltproduktion von Erdöl und Erdgas bescheiden bleiben, zumal kaum für den Export produziert werden wird. Trotzdem nimmt die Region eine Schlüsselstellung im globalen Kreislauf ein. Usbekistan, mit 25 Millionen Einwohnern bevölkerungsreichstes Land Zentralasiens, hat zudem eine geostrategische Position, die es jenseits der Energiereserven zu einem, wenn nicht zu dem Partner für Sicherheitspolitik in der Region werden lässt. Gerade hier spielen internationale WirtschaftsKonsortien eine wichtige Rolle. Investitionen, gerade in den Erdöl- und Erdgasmarkt, bedürfen politischer Situationen, die auch langfristig als gesichert gelten. An dieser Stelle übernehmen internationale Organisationen und Abkommen, wie der mit Aserbaidschan 1994 abgeschlossene »Jahrhundertvertrag « zur Ölförderung, eine geostrategisch stabilisierende Funktion. Im negativen Sinne bedeutet dies: Sicherheit vor Demokratie. Im positiven Sinne könnte es aber auch bedeuten: Sicherheit fördert Demokratie.

Usbekistan begibt sich durch die internationale wirtschaftliche Vernetzung, die zum einen nach Russland führt, zum anderen nach Korea und schließlich auch zu den USA in ein »System doppelter Machtallianzen «, aus denen nicht nur eine doppelte Abhängigkeit erwächst, sondern auch die gegenseitige Kontrolle der Allianzpartner übereinander. So entsteht ein sich selbstregulierendes System aus Interessenabhängigkeiten, das wiederum zur Stabilität der Region beitragen kann. Bisher jedoch hat Usbekistan wenig von dem eingelöst, was es im Selbstverständnis als Demokratie und als entsprechender Partner in eine Allianz einzubringen hätte. Wie in einer aktuellen Studie von HRW festgestellt wird, ist das Gegenteil der Fall: »Usbekistan ist ein enger Verbündeter der Vereinigten Staaten und anderer mächtiger westlicher Staaten, doch kann es sich nicht unter dem Deckmantel des Krieges gegen den Terror verstecken, um religiöse Unterdrückung zu rechtfertigen. [...] Die usbekische Regierung führt eine gnadenlose Kampagne gegen friedliche muslimische Andersdenkende. Das Ausmaß der Aktion und die Brutalität, mit der gegen unabhängige Muslime vorgegangen wird, zeigt ganz klar, dass wir es hier mit einer genau geplanten religiösen Verfolgung zu tun haben. «12

\footnotetext{
11 Vgl.: Energiecharta: www. europa.eu.int/scadplus/leg/de/lvb/127028.htm (Zugriff am 15. Dezember 2004).

12 Rachel Denber, Direktorin der Abteilung Europa und Zentralasien bei Human Rights Watch, vgl.: www.hrw.org/german/press/2004/ usbekistan033004de.htm (Zugriff am 09. November 2004)
} 
Die fünfjährige HRW-Studie zur religiösen Verfolgung in Usbekistan belegt anhand von Fallstudien und Interviews die politische Handlungsweise und Zielrichtung, mit der der usbekische Staatsapparat gegen jene »freien « Gläubigen vorgeht, die nicht gewillt sind, sich den streng vorgegebenen Richtlinien in der Religionsausübung zu unterwerfen:

»Es ist eine Schande, dass die internationale Gemeinschaft einer solchen Aktion tatenlos zusieht [...] Wenn die Verbündeten Usbekistans der Welt zeigen wollen, dass sie gegen die Verfolgung von muslimischen Andersdenken sind, müssen sie zu diesem Thema Stellung beziehen und dementsprechend handeln. «13

Bisher fand die Forderung von HRW an die USA und an europäische Staaten kein Gehör. Immerhin scheint die Menschenrechtssituation als Rahmenbedingung zunehmend in den Bewertungskatalog für Kredite der USA und der Europäischen Bank für Wiederaufbau und Entwicklung einzufließen, wenn auch nicht für die Bereitstellung von Krediten ausschlaggebend zu sein. Auch wenn die Europäische Bank überlegt, ihre Förderung einzustellen, liest sich das Statement des usbekischen Wirtschaftsministers betont optimistisch: »Im Finanzsektor besteht eine aktive Zusammenarbeit zwischen Usbekistan und der Eximbank der USA, die Kredite für die Finanzierung von 44 gemeinsamen Vorhaben über eine Summe von 1,5 Mrd. USD bereitstellte, sowie den größten amerikanischen Privatbanken wie J.P. Morgan Chase, Citigroup und anderen. Mit der führenden Versicherungsholding der USA ‘American International Group` sind zwei mit Erfolg arbeitende Versicherungsgesellschaften zur Absicherung von politischen und kommerziellen Risiken in London und Taschkent mit einem Kapital von mehr als 105 Mio. USD gebildet worden. So finden wir praktisch in allen Hauptbranchen führende amerikanische Unternehmen vor. Und es werden derzeit im Rahmen des Programms der Erweiterung und der Entwicklung der wirtschaftlichen Zusammenarbeit mit Geschäftskreisen der USA Dutzende von neuen perspektivischen Projekten in den Bereichen Erdöl/ Erdgas, Bergbauförderung, Entwicklung des Verkehrssystems, Telekommunikation, Informationstechnologien u.a. durchgearbeitet und zur Umsetzung vorbereitet. «14

\section{Die Angst vor dem »Dollar-Islam«}

Die Angst geht um in Usbekistan. Nicht die vor dem Kommunismus, hat dieser sich doch gegenüber dem »faulenden, parasitären und absterbenden « Kapitalismus weder als resistent noch als überlebensfähig erwiesen. ${ }^{15}$ Nein, es ist die Angst vor dem Islam. Nichts fürchtet die usbekische Führung so sehr, wie die Aktionen islamischer »Gerechter «, die im Namen Allahs einen wie auch immer gearteten Gottesstaat ausrufen wollen. Damit steht sie nicht alleine: Für Russland ist Usbekistan das »Nahe Ausland «, für Amerika Aufmarschgebiet im AntiTerror-Kampf. Russen, Amerikaner und Usbeken scheinen in trauter Runde versammelt. Alle eint die Furcht vor einem erstarkenden politischen Islamismus. Dem Berater des russi-

\footnotetext{
13 Ebd.

14 R. Azimov, stellvertretender Ministerpräsident und Wirtschaftsminister de Republik Usbekistan, vgl.: www.usbekistan.de.

15 Kommunistisches Manifest, erschienen erstmalig 1848.
}

schen Präsidenten, Pawlowskij, ist es dann auch lieber, wenn Amerikaner in Usbekistan sind, als Taliban in Tatarstan. ${ }^{16}$

Der politische Schulterschluss begann 1996, zu einem Zeitpunkt also, als sich die Taliban anschickten, ihr in Dörfern erprobtes Regime auf die Städte auszuweiten. Ihr Sieg über die politischen Widersacher verschaffte zumindest Kabul eine Atempause im seit 1979 nicht enden wollenden Bürgerkrieg. Eine religiöse Diktatur befriedete das Land, dessen Bevölkerung zu wählen hatte zwischen Krieg und Kommunismus oder Frieden und Islamismus. Es entschied sich mangels Alternativen für Letzteres. Und zog damit den Zorn Amerikas auf sich, das sich, einen Kreuzzug gegen religiösen Machtwahn postulierend, gegen den vermeintlichen Erzfeind der Demokratie, den Islam, in Position brachte. Der offensive Kampf gegen einen radikalen Islamismus fand in Russland und Usbekistan bereitwillige Anhänger: Russland, um damit an der kaukasischen Binnenfront erneut den als Anti-Terror-Kampf definierten Kolonialkrieg gegen Tschetschenien zu führen, Usbekistan aus einer als Islamphobie definierbaren Haltung. Ganz nebenbei förderte der US-amerikanische Aufmarsch beachtliche Millionen Dollar in die marode Staatskasse Usbekistans. Mehrere Faktoren unterstützten seit dem Zusammenbruch des Sowjetreiches auch in Usbekistan einen islamischen Aufschwung. Die inneren Faktoren wurden bestimmt durch die ambivalente Haltung der Regierung gegenüber den Spielarten des Islam. So fehlten Konzepte, wie die teils aggressiv vorgehenden islamistischen Gruppierungen in das politische System Usbekistans eingebaut werden konnten. Die Anfang der 1990er Jahre einsetzenden religiösen Missionierungsanstrengungen (im Übrigen nicht nur islamischer, sondern ebenso christlicher Organisationen) und der Talibanisierung Afghanistans stand die Regierung in Taschkent zu Anfang hilflos gegenüber. Zudem hatte die usbekische Regierung mit einem nachwirkenden Erbe der Sowjetzeit zu kämpfen. Über Jahrzehnte wurden islamische Bildungseinrichtungen vernachlässigt, oft zerstört und ihre Lehrer marginalisiert. Gleichzeitig hatten die sowjetischen Institutionen versucht, das in der Bevölkerung real existierende islamische Bewusstsein zu kanalisieren und staatlich zu instrumentalisieren. Nur noch zwei islamische Hochschulen gab es Ende der 1980er Jahre in Usbekistan. Die Zahl der Moscheen war auf kümmerliche 300 gesunken. ${ }^{17}$ Die Aufklärungsfunktion, die den religiösen Einrichtungen eben auch innewohnte, wurde damit weitgehend verhindert. Der Volksislam bzw. Brauchtumislam überlebte im privaten Bereich und vor allem in den für ihr festes Sozialgefüge bekannten Mahallas (Stadtvierteln) mit ihren traditionellen Strukturen. Keineswegs kann daher von einer Re-Islamisierung gesprochen werden, würde diese doch eine vorhergehende $D e$-Islamisierung voraussetzen. Allenfalls ließe sich von einer Re-Institutionalisierung des (Schrift-)Islam sprechen, der den zur Sowjetzeit gültigen staatlich verwalteten und damit kontrollierten Islam zu verdrängen suchte.

Die Implosion des Sowjetreiches hinterließ ein Machtvakuum, das auf religiösem Gebiet sehr schnell von Gruppen ausgefüllt

\footnotetext{
16 Zitiert nach: Halbach, Uwe: Islam und islamistische Bewegung in Zentralasien, in: Aus Politik und Zeitgeschichte, B 3-4/2002, S. 24

17 Den Geschichtsschreibern nach zu urteilen, durfte allein Buchara in seiner Blütezeit derart viele Moscheen sein Eigen nennen, dass Gläubige an jedem Tag des Jahres in einer anderen Moschee beten konnten.
} 
wurde, die durch ausländische Quellen finanziert und ideologisch geprägt wurden. Nun rächte sich das Fehlen eines innerusbekischen islamischen Netzwerkes, das die steigende Nachfrage der Bevölkerung an islamischen Themen hätte befriedigen können. Der fallende Eiserne Vorhang machte Anfang der 1990er Jahre den Weg frei für einen Islam-Import.

Je nach Provenienz förderten die Gelder und Missionsgesellschaften einen wahabitischen Islam (Saudi-Arabien) oder einen klassischen sunnitischen Islam (Ägypten). Die Folge war, in Verbindung mit der politischen und wirtschaftlichen Unzufriedenheit der Usbeken, ein starkes Anwachsen islamischer bis islamistischer Bewegungen. Die Regierung reagierte erst spät, ab 1995, mit einem eigenen Programm. So wurde schon 1995 ein internationales Zentrum für Islamforschung gegründet, dem 1997 die Islam-Universität Taschkent folgte. Ab 1998 ging die Regierung mit Gesetzesänderungen wieder restriktiv gegen die Gefahr religiöser Unterwanderung vor, die sich gegen islamische Äußerungsformen und ausländische Missionierungsbewegungen gleichermaßen richteten. Seitdem unterliegen alle religiösen Aktivitäten staatlicher Kontrolle, wurden nichtregistrierte Moscheen geschlossen (Schätzungen über Moscheen bewegen sich zwischen 6.000 und 20.000), Lautsprecher an Moscheen verboten und islamische Kleidung von Universitätsgeländen verbannt. ${ }^{18}$

Vor allem zwei Organisationen gilt die besondere Aufmerksamkeit des Staates. Es sind dies die Islamische Bewegung Usbekistans (IBU) und die Hizb ut-Tahrir al-Islami (HUT). Beide werden massiv bekämpft, auch wenn die politischen Zielsetzungen beider Gruppen voneinander abweichen. Während die IBU militant agiert und in Gewalt und Terror ein legitimes Mittel im Kampf gegen den Staat sieht, versucht die HUT, 1953 hervorgegangen aus einer Abspaltung der Moslembrüder, auf friedlichem Wege alle Muslime in einem Kalifat-Staat zu vereinen. Auch im Aufbau setzen beide Organisationen auf unterschiedliches Personal. Die IBU rekrutiert vornehmlich jugendliche Kampfeswillige aus dem Ferganatal. Dort hat sie auch ihre Rückzugsbasis. Von Washington auf die Liste der 2.000 gefährlichsten Terrororganisationen gesetzt, ist ihr militärisches Potential schwer einzuschätzen und liegt irgendwo zwischen 300 und 3.000 Kämpfern. ${ }^{19}$

Sehr viel nachhaltiger agiert die HUT. Nicht nur, weil sie Gewaltverzicht propagiert, sondern vor allem weil ihre Basis im urbanen und durchaus gebildeten Milieu gründet. Ihre geschätzten 10.000 Mitglieder agieren mit hoher Dynamik und ausschließlich auf ideologischem Gebiet. ${ }^{20}$ Ab 1998 stand in Usbekistan der Umgang mit freien islamischen Organisationen unter dem Banner der Vermeidung von Terrorismus. Als Hintergrund und Rechtfertigung für die staatlichen Maßnahmen werden in den Folgejahren jene bewaffneten Anschläge herangezogen, welche die IBU in den Jahren 1999 und 2000 in Usbekistan verübte. Die danach gestartete Kampagne gegen »islamischen Extremismus « traf und trifft aber weitaus weniger Extremisten, als vielmehr einfache Gläubige, die sich lediglich das Recht auf freie, d.h. individuelle, Glaubensausübung neh-

18 Vgl.: Hallbach, Uwe: 2002:31.

19 A.a.O.:29.

20 Institute for War and Peace Reporting: www. iwpr.net/index.pl?archive/ rca/rca_200108_64_1_eng.txt (Zugriff am 15. Dezember 2004). men. Selbst damit kollidieren sie mit staatlichen Religions- und Strafgesetzen, die - jenseits internationaler Standards - nur eine staatlich geleitete Religionsausübung zulassen.

Erschreckende Bestätigung und ideologische Unterstützung zog die usbekische Regierung aus den Anschlägen vom 11. September 2001. Seitdem wird im usbekischen Kampf gegen den Terror nicht selten zu direkter Gewalt und zu Folter gegriffen: »Beim Umgang mit unabhängigen Muslimen wenden usbekische Beamte systematisch Folter, Misshandlung, öffentliche Verleumdung an. $\ll^{21}$ Eine neue Anschlagsserie im März und April 2004 mit über 50 Opfern verstärkte die staatlichen Restriktionen gegen islamische Gläubige.

Den staatlichen Vorgaben stehen Organisationen wie Hizb ut-Tahrir und die Islamische Bewegung Usbekistan gegenüber, die sich in ihrem Bekenntnis zur Gewalt als Aktions- und Kampfmittel unterscheiden, nicht aber in ihrer ideologischen Ausrichtung. Die HRW-Studie spricht zudem der Hizb ut-Tahrir eine Gewaltlosigkeit zu, die den Eigendarstellungen nicht immer zu entsprechen scheint. »Die Ansichten unserer Organisation sind sehr radikal. Wir treten für den Sturz der bestehenden Regierungen in der gesamten muslimischen Welt und für die Errichtung eines islamischen Kalifats ein. Wir wollen dies gewaltlos und ohne Opfer erreichen, aber mit uns geht man wie mit Verbrechern um. In Wirklichkeit fürchten die Regierungen Zentralasiens, dass eine Oppositionspartei entsteht, die der Armut und der Korruption ein Ende setzen und somit den heutigen Machthabern die Basis entziehen kann. $\ll^{22}$

Während IBU offen gewaltbereit ist und daran keinen Zweifel aufkommen lässt, stellt sich Hizb ut-Tahrir als friedliebende und vor allem Gewalt ablehnende Organisation dar. Ein genauerer Blick auf die Homepage gibt aber ein Szenario frei, das in seiner politischen Zielsetzung keinen Zweifel an der Demokratiefeindlichkeit der HUT lässt. »Hizb ut-Tahrir hat die Notwendigkeit erkannt, für die Vernichtung der Systeme des Kufr zu arbeiten [...] Jedoch hat der Präsident Usbekistans Karimov, dieser ungläubige Jude und sein verbrecherisches System, in den letzten beiden Jahren einen üblen Krieg gegen die Parteimitglieder in Usbekistan gestartet $«{ }^{23}$ Dem Kampf gegen das System wird alles untergeordnet, ja, das Leben selbst wird zum Kampfmittel. Indem »die Wiederaufnahme des islamischen Lebens und die Wiedererrichtung des Kalifats « zur »Schicksalsfrage « erklärt werden, sei es eine »Frage auf Leben und Tod, für deren Sache Menschenleben von geringer Bedeutung sind. ${ }^{24}$ Kaum deutlicher könnte man die Opferbereitschaft der Mitglieder charakterisieren. Fast schon als Aufruf zur Gewalt liest sich indes die folgende Passage: »Gebt euer Vermögen und euer Leben auf dem Wege Allahs, seid tapfer und mutig und bewahrt immer stark und lebendig eure Aqida [Glaubensüberzeugung, H.F.] in euren Seelen.» ${ }^{25}$ Umso erstaunlicher ist es daher, dass HRW in der Studie zu

\footnotetext{
21 Staatsfeinde schaffen: Religiöse Verfolgung in Usbekistan. HRW-Studie, vgl. www.hrw.org.

22 Statement einer als von der Deutschen Welle als Aktivistin der Hizb utTahrir bezeichneten Gesprächspartnerin. Vgl.: www.dw-world.de/dwelle/ cda/detail/dwelle.cda.detail.artikel (Zugriff am 09.11.2004).

$23 \mathrm{Vgl}$.: www.hizb-ut-tahrir.org/deutsch/leaflets/wilayatlflts/Usbekistan/ shabab_Usbekistan_Rede.htm (Zugriff am 09.11.2004)

24 Ebd.

25 Ebd.
} 
dem Ergebnis kommt, dass es sich bei Hizb ut-Tahrir um eine Organisation handele, die Gewaltverzicht propagiere.

Die »Unabhängige Organisation für Menschenrechte Usbekistan« sprach 2001 von 7.600 politischen Gefangenen, von denen 7.400 religiösen Organisationen zuzurechnen seien. Im Jahresbericht 2003 von Human Rights Watch werden 6.000 politische Gefangene angegeben, die meisten wegen »friedlicher Äußerungen ihrer religiösen Gesinnung «. ${ }^{26}$ Dies zeigt zweierlei: Zum einen hat die Regierung, trotz aller Beteuerungen, kaum Abstand genommen von ihrer repressiven Politik der oppositionellen Ausgrenzung. Zum anderen scheint ein politischer Maßnahmenkatalog aus Bildung und Aufklärung zu wirken. Mit Hilfe eines Bildungswesens, das eine neunjährige Schulpflicht vorsieht, versucht der Staat, den religiösen Organisationen zu begegnen.

Mit den Unruhen, die im Mai 2005 in Andijan, einer Großstadt im Fergana-Tal, ausbrachen, kehrte das Karimov-Regime zu seinen Wurzeln zurück. In dem scheinbar gut organisierten Aufstand, weltweit verstanden und interpretiert als Zeichen wirtschaftlicher und sozialer Missstände in der Region, sieht die Regierung ein islamistisches Umsturzszenario, das mit aller staatlicher Machtfülle im Blute ertränkt wurde. Genaue Zahlen über Tote und Verletzte liegen nicht vor, können auch gar nicht vorliegen, würde dies doch eine transparente und von staatlichen Institutionen gewollte Aufarbeitung bedeuten. So gehen die Angaben von Beobachtern von bis zu 500 Toten, mehreren tausend Verletzten und Tausenden Flüchtlingen aus. Die von der Karimov-Regierung beschworene islamistische Bedrohung, die es zu zerschlagen galt, sei von der gewaltbereiten AkramijaBewegung, benannt nach dem religiös-politischen Aktivisten Akram Yoldaschew, ausgegangen. Dabei handelt es sich um eine Organisation, die bis zu den Mai-Unruhen weitgehend unbekannt war, und bei der es sich um einen lokalen Ableger der HUT handeln soll. Der Initiator Yoldaschew trat schon Anfang der 1990er Jahre mit religiöser Propaganda auf und wurde 1999 unter dem Vorwurf des Terrorismus verhaftet.

Das Vorgehen im Stile einer autokratisch-absolutistischen Herrschaft beweist zweierlei: Zum einen die Grenzen eines Demokratie-Exportes. Auch wenn in Georgien, in der Ukraine und in Kirgistan bedeutende Schritte im Systemwechsel von FSU-Staaten erfolgten, ist dies nicht der Königsweg hin zu demokratischen Strukturen. In Usbekistan, einem Land mit weitgehend intakten regionalen Clan- und Machteliten, ist deren Beharrungsvermögen groß. Unberührt von internationalen Protesten wissen sie ihre Pfründe zu verteidigen.

Da sich zunehmend internationale Organisationen aus Usbekistan zurückziehen, verkümmert der Prozess der Demokratisierung immer mehr zum Feigenblatt einer sich demokratisch gerierenden, gleichwohl Grundstandards wie Religions- und Meinungsfreiheit missachtenden Diktatur. So hätten, nach Aussagen des Chefredakteurs der InternetZeitschrift Tribune-UZ, Alo Hocajew, zum internationalen Tag der Presse in Taschkent lediglich die Friedrich-Ebert-Stiftung und die Konrad-Adenauer-Stiftung Veranstaltungen organisiert. ${ }^{27}$

\footnotetext{
26 Vgl.: www. hrw.org/english/docs/2003/12/31/uzbeki7024.htm (Zugriff am 27. Januar 2004).

27 Siehe Interview mit Hocajew in der Zeit, 02. Juni 2005.
}

Zum anderen fördert die politische Gleichschaltung des Landes andere Arten oppositioneller Meinungsbildung und Willensäußerung, die u.a. im islamischen Milieu auf eine existierende Infrastruktur zurückgreifen kann.

\section{Fazit}

Hinter dem Kampf gegen islamische Organisationen steht ein politisches Motiv: Besitzstandswahrung und Machterhalt. So sieht sich das politische Regime unter Karimov im Konkurrenzkampf mit Organisationen wie Hizb ut-Tahrir oder IBU. Und in der Tat scheinen HUT und IBU einen politische Islam zu propagieren, der das Ende des staatlichen Systems ebenso einfordert wie die Einführung des Kalifats und islamischer Rechtsprechung. So kann Karimov unter Verweis auf staatsfeindlichen islamischen Extremismus auch jene charismatischen islamischen Führer bekämpfen, die weder politisch inspiriert sind noch einen Systemwechsel auch nur propagierten. Damit aber bekämpft der Präsident gerade jene potentiellen Partner, die ihm als Multiplikatoren und Konfliktschlichter im Kampf gegen den politischen Islamismus und Terrorismus zur Seite stehen könnten. Hier greift die restriktive Politik des Staates nicht nur zu kurz, sie zeigt sich kontraproduktiv. Indem Gläubige an den Rand des Extremismus gedrängt werden, einzig weil sie »zu fromm« seien oder Bärte trügen, nähern sich diese auch jenen extremen Positionen an, die vorher keine Rolle im religiösen Leben gespielt haben. Bei Geiselnahmen spricht man gerne vom »Helsinki-Syndrom «, um zu verdeutlichen, wie sich Opfer und Täter im Angesicht einer externen Gefahr (Polizei, SEKH) zu solidarisieren beginnen. In Anlehnung daran kann die Verfolgung von islamischen Gläubigen als Karimov-Syndrom bezeichnet werden.

Ob eine langfristige politische Demokratisierung möglich wird, ist im hohen Maße an die sozioökonomische Entwicklung des Landes gekoppelt. Noch fehlt es der Regierung an ausreichendem Reformwillen, um dem Land die nötige Rechts- und Investitionssicherheit zu geben. 